\title{
A New Burst Scheduling Algorithm for Edge/Core Node Combined Optical Burst Switched Networks
}

\author{
SeoungYoung Lee, InYong Hwang, and HongShik Park \\ $\mathrm{BcN}$ Engineering Research Center, Information and Communications University, \\ 103-6 Munji-Dong, Yuseong-gu, Daejeon, Korea \\ \{seoungyoung, iyhwang, hspark\}@icu.ac.kr
}

\begin{abstract}
The burst contention problem in Optical Burst Switching network is an intrinsically serious problem. Many researches have tried to solve this problem, however it have been known that avoiding the burst loss is very difficult issues in the current OBS network. To improve burst blocking rate, we consider the edge/core combined OBS network where the core node performs the edge node function as well. Through this architecture, available amount of data burst that the node generates can be expected with respect to offset-time of transit data bursts. Any researches for this area has not been performed, thus we propose a new data scheduling algorithm for the edge/core combined OBS network where data bursts that the node generates do not interrupt transit data bursts from previous nodes. We analyzed the data burst loss rate and the throughput in relation with the offset-time of transit data bursts. Results show that the loss rate of the data bursts is drastically reduced and the throughput improves when the offset-time of transit data bursts increases.
\end{abstract}

Keywords: OBS, JET, scheduling, void filling, CoS.

\section{Introduction}

The emergence of wavelength division multiplexing (WDM) technology is considered as a solution to fulfill the tremendously increasing demands of transmission bandwidth driven by the growth of IP-based data traffic. At the same time, the necessity to make the next-generation optical Internet architecture is augmented, which can transport IP packets directly over the optical layer without opto-electro-optic (O/E/O) conversions, like optical packet switching (OPS). Although OPS which can achieve higher utilization is attractive, there are practical limitations such as optical buffer and all optical processing. Presently, optical burst switching (OBS) technology is under study as a solution for optical Internet backbone in the near future since OBS technology can cut through data messages without $\mathrm{O} / \mathrm{E} / \mathrm{O}$ conversion and guarantee the Class of Service $(\mathrm{CoS})$ without any buffering [1-2].

The analyses of OBS have been focused on the ring network or simply mesh network. Recently, because the issues about the commercialization have been increased, the studies for the real networks, especially for the mesh-type networks, have been increased [3-4]. In this paper, to meet the research trend of OBS network, we consider the edge/core node combined (ECNC) OBS network, where all node can generate data burst (DB) with the edge node function and forward DB to the next node with the core node function. If the node control the sending time of its DB by buffering the DB 
in the electrical buffer, it will not interrupt the transit data bursts (TDB) generated previous nodes. Therefore, the starting DB can avoid contention by inserting DB among TDB. By doing so, the overall network performance will be improved while this scheme do not affect the loss rate of TDB. We find that the offset-time of TDB affects the throughput of the network and analyze throughput mathematically. The remainder of paper has been structured as follows. Section 2 reviews the burst blocking probability in conventional OBS network. Section 3 presents the new scheduling algorithm suitable for the ECNC OBS network. Section 4 provides the analysis results, and the conclusion follows in Section 5.

\section{Blocking Probability in OBS Network}

\subsection{Network Modeling in Conventional OBS Network}

In Figure 1, the typical network model in the conventional JET OBS network is shown. In this model, the ingress node 1, 2, and 3 send data bursts to the egress node 5 through the core node 4, respectively. Data bursts from the three ingress nodes should contend for the same resource at the output port of the core node 4 . If we assume that data bursts are arriving at a bottleneck node with Poisson distribution and the number of channel is $\mathrm{k}$ and traffic loads for node 1,2 and 3 are $\rho_{1}, \rho_{2}, \rho_{3}$, respectively, then the burst blocking probability with no buffer can be calculated by using the well-known Erlang loss formula $P_{B}\left(k, \rho_{1}+\rho_{2}+\rho_{3}\right)$. But, in the mesh-type networks, the blocking rate will be changed because of the edge/core node combined functions.

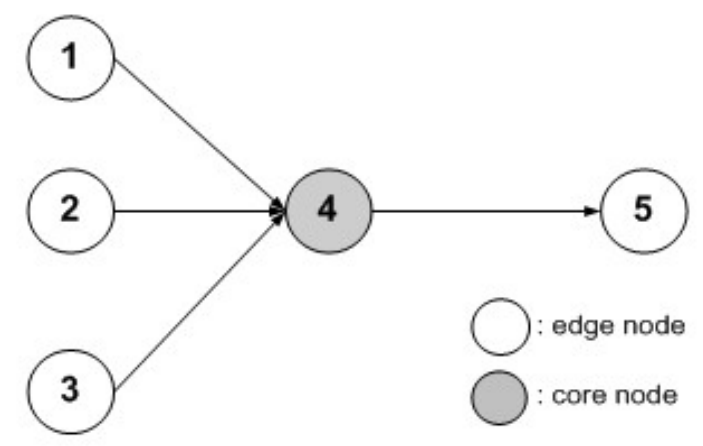

Fig. 1. Network model in the conventional OBS network

\subsection{Network Modeling in ECNC OBS Network}

In this section, we investigate the performance of the ECNC OBS network as depicted in Figure 2. In Figure 2 (a), the edge/core node combined, the node 4, performs the egress function as well as the core function. 


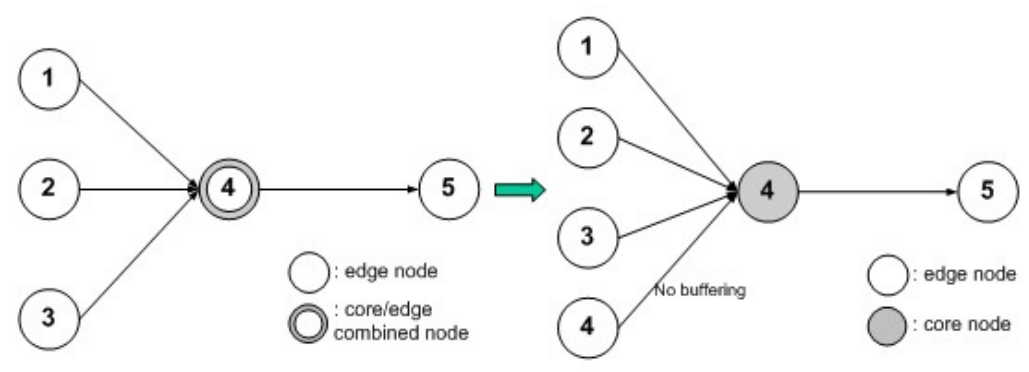

(a) Physical model

(b) Logical model

Fig. 2. Network model in the ECNC OBS networks

Thus, it both generates new bursts as an ingress node and cut-though bursts from ingress nodes as the core node. Depicted as the logical model in Figure 2 (b), data bursts from 4 also contend for the outgoing port. Thus, burst blocking probability can be calculated by $P_{B}\left(k, \rho_{1}+\rho_{2}+\rho_{3}+\rho_{4}\right)$. It is noted that the node 4 do not have buffers. Instead, self-generated data bursts are immediately sent to the outgoing port and contend with TDB after assembled by using the conventional burst assembly schemes [5].

\section{Proposed Channel Scheduling Algorithm}

\subsection{New Scheduling Algorithm for ECNC OBS Network}

In the previous chapter, we know that node 4 has the capability to buffer self-generated data bursts for the purpose of void filling between TDB. We propose a new scheduling algorithm for ECNC OBS network to improving throughput as well as reducing the data burst loss rate. In Figure 3, data bursts from 3 ingress nodes contend for the outgoing port of the node 4 , however data bursts generated from the node 4 do not contend with TDB

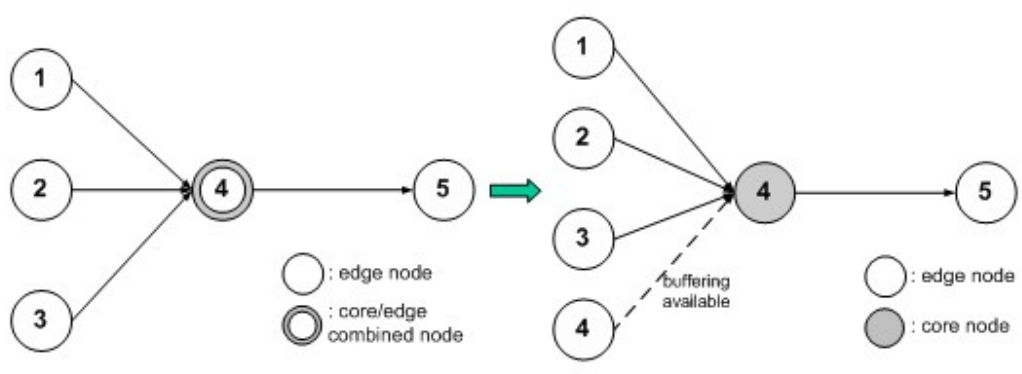

(a) Physical model

(b) Logical model

Fig. 3. Network model for the new data scheduling algorithm in edge/core node combined OBS network 
but fill void intervals. This void-filling is based on the two capabilities of the node 4 , one is the monitoring capability for all voids in the data channel scheduling table and the other is buffering capability to shift data bursts generated by node 4 . Thus, the burst blocking probability for all inputs $\left(\rho_{1}, \rho_{2}, \rho_{3}\right.$, and $\left.\rho_{4}\right)$ can be calculated by $P_{B}\left(k, \rho_{1}+\rho_{2}+\rho_{3}\right)$ where $\rho_{4}$ has no impact on the loss probability. Compared to loss probability with conventional schemes, $P_{B}\left(k, \rho_{1}+\rho_{2}+\rho_{3}+\rho_{4}\right)$, the proposed scheduling scheme achieves drastic loss rate reduction for data bursts.

\subsection{Throughput of Edge/Core Node Combined Network}

To increase throughput, the node uses the void intervals between TDB to transmit one-hop-going (OHG) data bursts. The average burst size of OHG data bursts can be driven by using the analytical method for the proposed network topology. The mean available size of the data burst is the function of the offset-time and the traffic load of the TDB as shown in equation (1) when the offset time of TDB is constant [6].

$$
\overline{h_{\text {in }}}=f\left(t_{\text {offset }}, \rho_{c}^{\text {each }}\right)=\int_{0}^{t_{\text {offset }}} x \cdot p(x) d x+\int_{t_{\text {offset }}^{\infty}}^{\infty} t_{\text {offset }} \cdot p(x) d x
$$

Where, $t_{\text {offset }}$ is the offset time of TDB, $\rho_{c}^{\text {each }}$ is traffic load of TDB per each channel, $\mathrm{p}(\mathrm{x})$ is the probability that void interval is $\mathrm{x}, \mathrm{x}$ is the length of void interval, respectively. By using this size of self-generated DB, the throughput of the channel can be calculated analytically with the function of offset-time and traffic load of TDB.

\section{Numerical Results}

Performance is analyzed based on Figure 2 and Figure 3 for the conventional scheduling algorithm and our proposed data channel scheduling algorithm. Three ingress node and one core node generate data bursts equivalently with Poisson distribution. We assume the data burst size from ingress nodes is same for simplicity. All links consist of 8 wavelengths.

In Figure 4, the burst blocking rate comparisons between the conventional and our proposed data channel scheduling algorithm are presented for the edge/core combined OBS network. It is clearly shown that proposed algorithm has better performance than conventional algorithm because self-generated data bursts do not affect the overall burst blocking rate. Therefore, it is possible to save overall resources to be provided for guaranteeing a certain level of the blocking probability.

To investigate the relationship between offset time TDB and throughput, we compare the throughput of core node by changing the offset time. In Figure 5, while the throughput improvements do not appear in conventional method, the throughput of proposed scheme increases when the offset time ratio to the mean length of TDB changes from 0.2 to 1.0. Throughputs of Figure 5 are acquired by using the equation (1) and the termination rate of void interval of TDB [6]. It means that the throughput will be improved if the offset time of TDB increases for the proposed scheme at the same traffic load. 


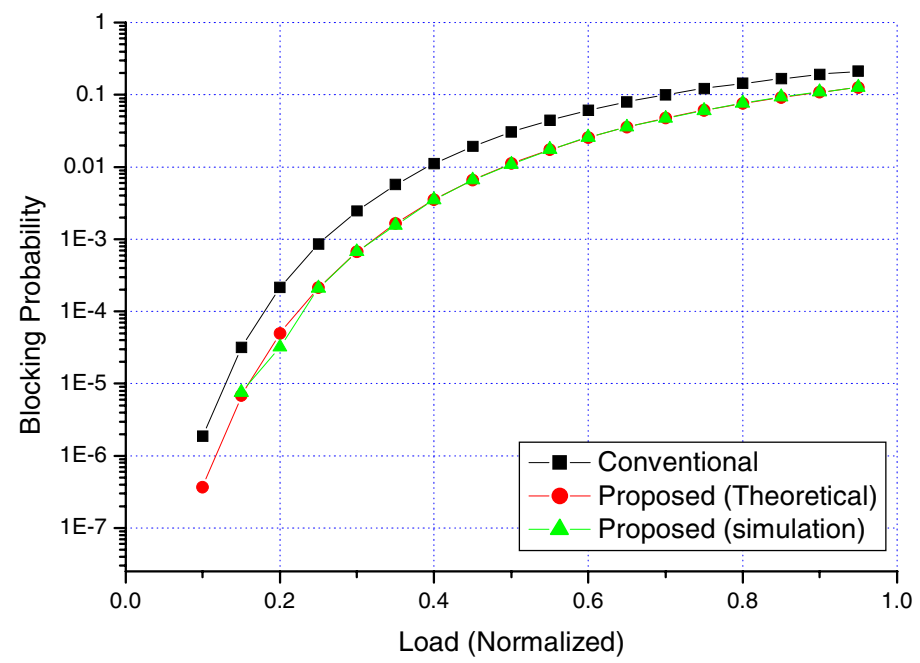

Fig. 4. Data burst blocking rate in conventional and proposed data burst scheduling algorithm in the edge/core combined OBS network

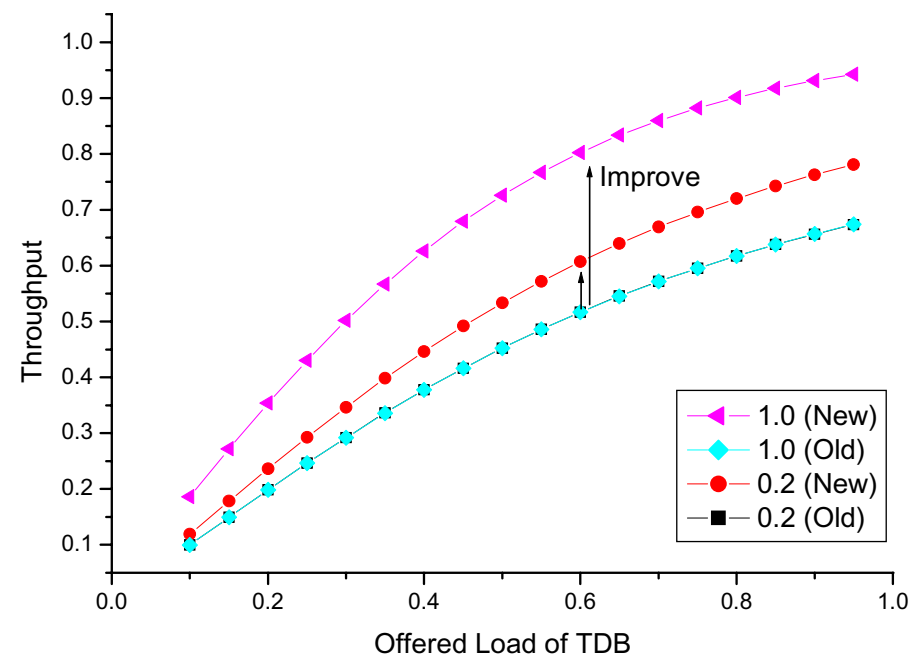

Fig. 5. Relationships between offset-time and throughput

\section{Conclusion}

In this paper, we propose a new data channel scheduling algorithm for the core/edge node combined OBS network to utilize buffering effect in the ECNC node. If we consider that the nodes perform the edge and core node function, the conventional data channel scheduling algorithm should be modified. Without new scheduling scheme, we cannot achieve any merit of the ECNC OBS nodes. In our proposed data 
channel scheduling algorithm, the self-generated data bursts at edge/core combined node do not contend with the TDB by using the void intervals and electrical buffering function. Through performance analysis results, it is clear that our proposed scheduling algorithm reduces data burst loss probability and acquires high channel utilization and great benefit to the performance of mesh-type networks.

\section{Acknowledgements}

This work was supported in part by the Institute of Information Technology Assessment (IITA) through the Ministry of Information and Communication (MIC) and the Korea Science and Engineering Foundation (KOSEF) through the Ministry of Science and Technology (MOST), Korea.

\section{References}

1. M. Yoo and C. Qiao.: A new Optical Burst Switching Protocol for Supporting Quality of Service. Proc. SPIE All Optical Comm. Syst.: Architecture, Control Network Issues, Vol. 3531, 1998, pp.395-405.

2. Qiao, C.: Labled Optical Burst Switching for IP-over-WDM Integration. IEEE Communication Magazine, Vol.1, No. 9, September 2000, pp. 104-114.

3. Fei Xue, S.J.B. Yoo, H. Yokoyama and Y. Horiuchi.: Performance comparison of optical burst and circuit switched networks. Optical Fiber Communication Conference, Vol 3, March 2005.

4. X. Huang, V.M. Vokkarane and J.P. Jue.: Burst cloning: a proactive scheme to reduce data loss in optical burst-switched networks. International Conference on Communications, Vol 3, May 2005, pp. $1673-1677$

5. Y. Xiong, M. Vandenhoute, H. Cankaya.:Control architecture in optical burst-switched WDM networks. IEEE JSAC, Vol. 18, 2003, pp. 1838-1851.

6. S.Y. Lee, I.Y. Hwang and H.S. Park.:A New Burst Generation and Scheduling Scheme in Optical Burst Switching Networks. submitted to IEICE 